\title{
All-Silicone-Based Distributed Bragg Reflectors for Efficient Flexible Luminescent Solar Concentrators
}

\author{
Mark Portnoi ${ }^{\mathrm{a}}$, Thomas J Macdonald ${ }^{\mathrm{b}, \mathrm{c}}$, Christian Sol $^{\mathrm{a}}$, Tom S Robbins ${ }^{\mathrm{a}}$, Tao Li ${ }^{\mathrm{a}}$, Johannes Schlaefer ${ }^{\mathrm{a}}$, Stefan Guldin ${ }^{\mathrm{d}}$, Ivan P \\ Parkin $^{\mathrm{b}}$, Ioannis Papakonstantinou ${ }^{\mathrm{a}, *}$ \\ ${ }^{a}$ Photonic Innovations Lab, Department of Electrical Engineering, University College London, London, WC1E 7JE, website: https://www.ee.ucl.ac.uk/pilab \\ ${ }^{b}$ Department of Chemistry, University College London, London, WC1H OAJ \\ ${ }^{c}$ Department of Chemistry, Imperial College London, London, W12 OBZ \\ ${ }^{d}$ Department of Chemical Engineering, University College London, London, WC1E 7JE
}

\begin{abstract}
Luminescent Solar Concentrators (LSCs) have drawn huge interest recently as a technology to pave the way towards the seamless integration of photovoltaics to a range of high-value industries; from architecture and sports to leisure and consumer electronics. Additional device flexibility comes with the inherent ability to attain freeform shapes, expanding the possible fabrication methods, applications and retro-fitting techniques. Unfortunately, flexible LSCs suffer from curvature induced losses which can severely reduce their efficiency, inhibiting the potential of large-scale devices. In this work, we experimentally demonstrate an all-silicone based flexible LSC and Distributed Bragg Reflector (DBR) combination diminishing curvature induced losses. The DBRs, fabricated using scalable solution-based processes, exhibit optical properties precisely engineered to partner our LSCs, as well as high uniformity, resistance to temperature and curvature. Comprehensive modelling shows that for large-scale devices $\left(1 \mathrm{~m}^{2}\right) \mathrm{we} \mathrm{can}$ essentially decouple the performance of the LSC from curvature, steering the technology towards commercial viability.
\end{abstract}

Keywords: Luminescent Solar Concentrators, Solar Energy, Distributed Bragg Reflectors, Flexible Devices

\section{Introduction}

Flexible Luminescent Solar Concentrators (LSCs) have attracted attention as an opportunity to bring integrated photovoltaics to a range of industries from architecture to consumer electronics and leisure $[1,2,3,4,5,6,7,8,9]$. Flexibility also brings the opportunity of roll-to-roll manufacturing, leading to decreased production costs and retrofitting of existing windows and facades $[10,11]$.

In general, LSCs are composed of transparent polymer sheets doped with randomly oriented luminescent particles, known as fluorophores. The fluorophores absorb incident sunlight, and re-emit it isotropically at longer wavelengths. A portion of the reemitted sunlight is trapped by means of total internal reflection and guided to the edges, where it can be coupled into solar cells and converted into electricity, as shown in Figure 1a. Due to the energy shift between absorbed and emitted light, LSCs can collect light from a larger acceptance angle than conventional solar cells, and can in theory concentrate light to small areas extremely efficiently $[12,13]$. The broad freedom of design in colour, transparency, shape and rigidity, makes the devices an ideal PV-module candidate for integration into our everyday lives.

Unfortunately, LSCs are subject to two main sources of loss that have hindered their commercial deployment so far; quan-

\footnotetext{
*Corresponding author, e-mail: i.papakonstantinou@ucl.ac.uk (c)2020. This manuscript version is made available under the CC-BY-NC-ND 4.0 license http://creativecommons.org/licenses/by-nc-nd/4.0/
}

tum yield and escape cone losses. Quantum yield losses are caused due to a fraction of the absorbed light not being reemitted by the fluorophores and being lost as heat. Recent advances in new luminescent materials have led to fluorophores with near-unity quantum yields and low re-absorption, including core-shell nanocrystals [14, 15, 16, 17], silicon quantum dots $[9,18]$, perovskite quantum-dots $[19,20,21]$, and quantumcutting technologies [22].

Whilst significant progress in reducing quantum yield losses has been made, escape cone losses still remain a major barrier particularly for larger area and flexible devices. In this case, light is either re-emitted or scattered in a direction such that it falls upon the interfaces at an angle smaller than the critical angle of the host matrix, resulting in the light escaping the larger faces of the device. The critical angle, $\theta_{C}$, is given by Snell's law and can be calculated by $\theta_{C}=\sin ^{-1}(1 / n)$ where $\mathrm{n}$ is the host matrix refractive index [23]. For typical waveguides made of polymethyl methacrylate (PMMA), the escape cone losses of flat LSCs, with no re-absorption losses or additional sources of scattering, are $\approx 25 \%$. This can be significantly increased in the presence of fluorophore reabsorption, host matrix absorption and scattering [12].

When LSCs are bent, the introduced curvature can contribute additional losses of over $10 \%$ [6, 24]. These comprise of both aggravated quantum yield losses, due to longer path lengths, and escape cone losses due to a decrease in waveguiding efficiency. The curvature induced losses are particularly pronounced in the case of materials with overlap in absorption 

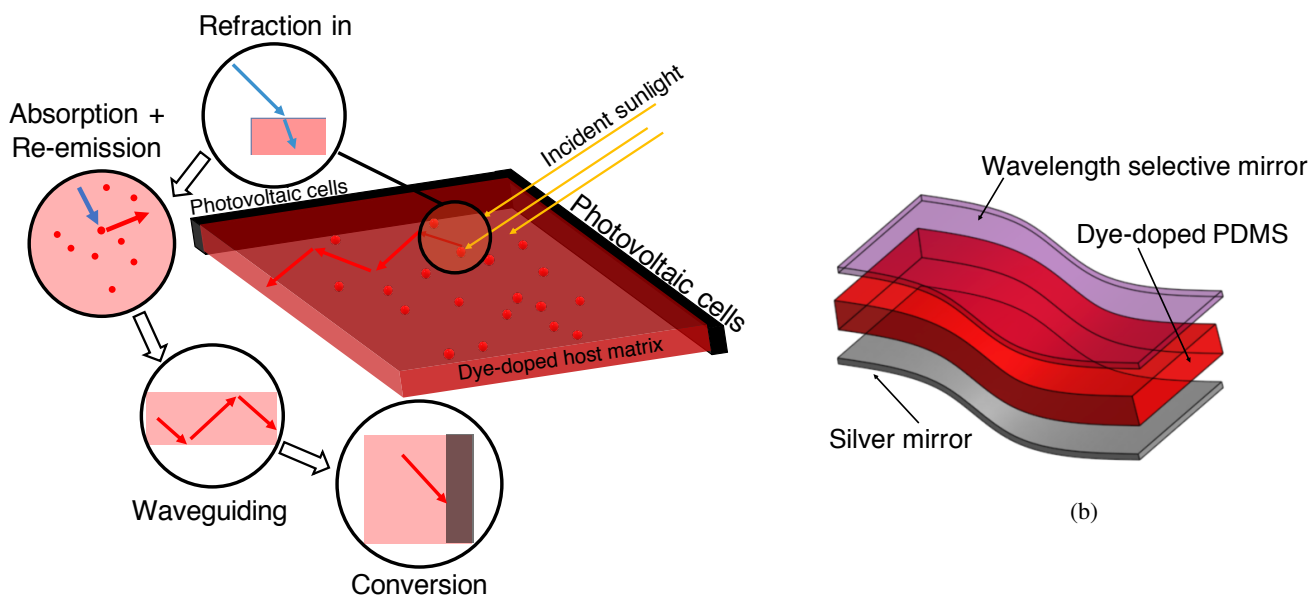

(b)

(a)
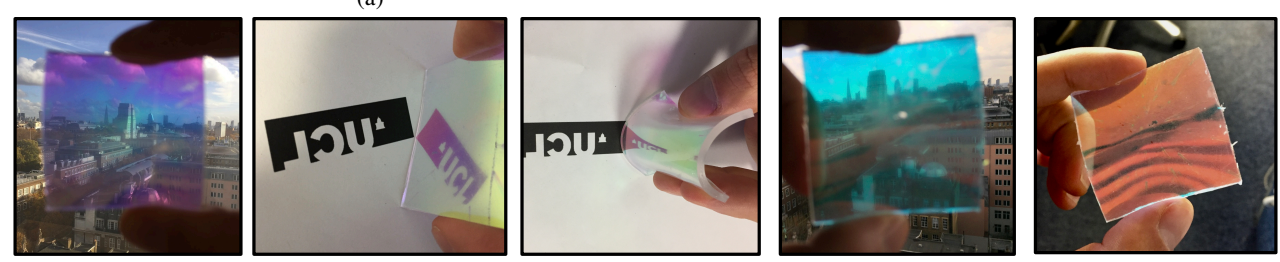

(c)

Figure 1: a) Diagram showing the principle of operation of luminescent solar concentrators. b) Diagram of device configuration (from top to bottom): wavelength selective mirror, dye-doped PDMS and silver mirror. c) Photographs of two fabricated flexible wavelength selective mirrors. The device with peak reflectance at $605 \mathrm{~nm}$ in transmission (far-left) and in reflection (left and centre). A second device with peak reflectance at $670 \mathrm{~nm}$ in transmission (right) and reflection (far-right).

and emission spectra and can pose a significant barrier to viable flexible devices [24].

While in the case of flat LSCs several photonics based solutions have emerged in an attempt to curb escape cone losses, never before has there been an effort to mitigate the extra losses in flexible devices. In the rigid case, one of the most prominent solutions is to use wavelength selective mirrors to increase the waveguiding efficiency (known also as internal optical efficiency and more rigorously defined in the methods section). In the context of LSCs, wavelength selective mirrors are designed to be transmissive in the absorption range of the chosen fluorophore, whilst selectively reflecting light in the wavelength range of its emission. The result is that even light that falls within the escape cone of the host matrix is kept within the LSC, boosting the internal optical efficiency. There are currently several designs for rigid configurations. In one example, authors suggested the use of cholesteric liquid crystals [25, 26]. Another promising wavelength selective mirror technology is the application of Distributed Bragg Reflectors (DBRs), which leverage thin-film interference effects to engineer suitable passbands and stopbands in their optical response [27]. Their potential has been explored theoretically [28, 29, 30], and demonstrated experimentally with spin-coated $\mathrm{SiO}_{2} / \mathrm{SnO}_{2}$ DBRs on dye-doped LSCs [31], and with DBRs on CdSe/CdS quantum dot doped LSCs [32]. There has also been a demonstration of a curved DBR, but this was applied to a flat, rigid, PMMA LSC which could not be deformed or reshaped [33].

In this paper we demonstrate a unique, all-flexible LSC-DBR design that allows for virtually any freeform LSC shape to be achieved. Both LSC and DBR systems consist entirely of silicone-based materials, resulting in mechanical consistency and compatibility throughout the device. Because of the elastomeric properties and compatibility of the chosen materials, we show that the device can be repeatedly deformed and reshaped without its performance being affected. We exhibit excellent control of the optical properties of our DBRs by engineering both the thin-film thickness and refractive index contrast, allowing us to tailor their properties precisely to partner our LSCs. With an eye to future scaling up, the DBRs, pictured in Figure 1c, are fabricated using industrially available, singlepot solution-based processes. Upon integration with an LSC, as shown in Figure 1b, we demonstrate a reduction of device escape cone losses by a quarter, as well as a significant reduction of efficiency dependence on curvature. Furthermore we analyse the performance of large scale LSCs by using Monte-Carlo statistical models demonstrating a clear pathway to real consumer products.

\section{Results and discussion}

\subsection{Thin film materials}

To obtain flexible DBRs with high peak reflectance we sought to use two materials with a series of requirements. We aimed for the optical properties of the materials to provide high refractive index contrast, low absorption and low scattering. The materials should also have good adhesion and mechanical compatibility, alongside flexibility. 


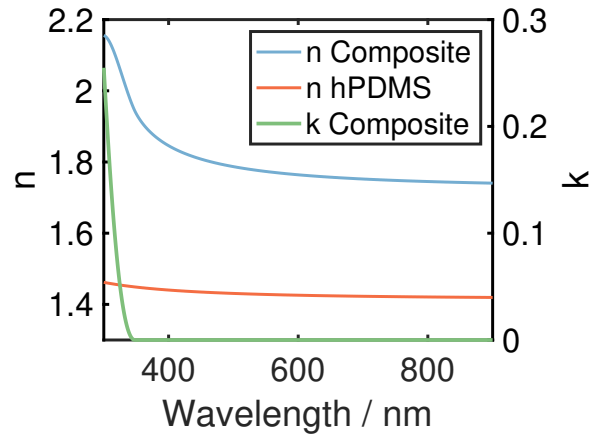

(a)

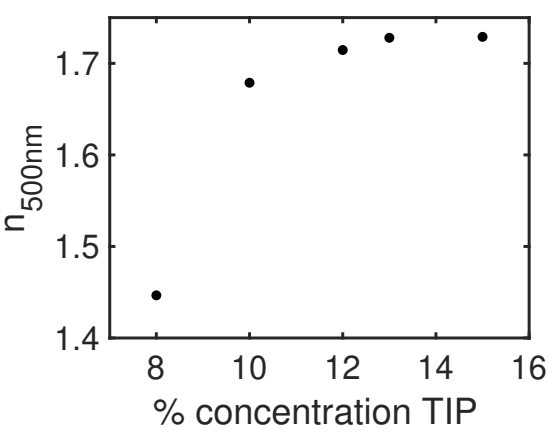

(b)

Figure 2: a) Real (n) and imaginary (k) parts of the refractive indices measured using spectroscopic ellipsometry. Y-axis scales for $\mathrm{n}$ on left and $\mathrm{k}$ on right. b) Refractive index of the $\mathrm{TiO}_{2}$ PDMS composite at $500 \mathrm{~nm}$ as a function of TIP loading concentration by weight percentage of total solution.

Our flexible DBRs are composed of alternate layers of two polydimethylsiloxane (PDMS) based materials with refractive index contrast $\Delta n>0.3$ across the entire visible spectrum. For comparison, optical fibres have typical $\Delta n \approx 0.02$ [34]. The refractive indices, shown in Figure 2a, were measured using spectroscopic ellipsometry. The fitting models for the materials used to derive the optical constants shows excellent agreement $\left(R^{2}>0.99\right)$, and are shown in the Supplementary Materials Section 1.1. The fabrication process is a scalable, solution based sol-gel spin coating technique. Recipes for the fabrication of both solutions and the devices can be found in the methods section.

The lower refractive index material $\left(\mathrm{n}_{500 \mathrm{~nm}} \approx 1.4\right)$, is a commercially available silicone, hPDMS (GELEST). We chose this material for its high optical transparency $(\mathrm{k} \approx 0$ for $300-900$ $\mathrm{nm}$ ) and highly uniform thin films.

The higher refractive index material $\left(\mathrm{n}_{500 \mathrm{~nm}}>1.7\right)$ is a titanium dioxide $\left(\mathrm{TiO}_{2}\right)$ - PDMS composite synthesised in our laboratory, in which $\mathrm{TiO}_{2}$ nanoparticles act as crosslinkers for PDMS, as shown in previous studies [35]. For the composite synthesis, titanium (IV) isopropoxide (TIP) and hydroxyterminated polydimethylsiloxane (PDMS-OH) are used as precursors. Acetic acid is used to control hydrolysis, limiting the size of $\mathrm{TiO}_{2}$ particles within the composite. A small amount of surfactant (BYK-378) is used to reduce surface tension in the thin films and to allow for better wetting during spin coating.

The $\mathrm{TiO}_{2}$ nanoparticles in the composite act as crosslinkers for the hydroxy terminated PDMS. This was confirmed by the presence of Ti-O-Si bonds in fourier-transform infrared spectroscopy (FTIR) and X-ray photoelectron spectroscopy (XPS) measurements as described in the Supplementary Materials Sections 1.2 and 1.3.

The optical properties of the composite can be controlled significantly. We have demonstrated control of the refractive index by varying the concentration titanium (IV) isopropoxide precursor. To demonstrate this we fabricated a series of samples with increasing TIP concentrations. As shown in Figure 2b, the composite's refractive index increases with increased loading of TIP. We further verify and discuss the surface chemistry of the $\mathrm{TiO}_{2}$-PDMS composite with further XPS measurements, as shown in the Supplementary Materials Section 1.3.

When optimising the composite we considered the compromise between the higher refractive index contrast associated with increased $\mathrm{TiO}_{2}$ loading and the polymeric properties of the PDMS. Increased $\mathrm{TiO}_{2}$ content results in reducing the adhesion and mechanical flexibility of the film. Simultaneously, scattering and absorption are increased as they are both particle size and concentration dependent properties [36].

Transmission electron microscopy (TEM) images of the $\mathrm{TiO}_{2}$-PDMS composite were taken, showing the presence of both rutile and anatase $\mathrm{TiO}_{2}$ nanoparticles with a size of $\approx 5$ $\mathrm{nm}$. The observed $5 \mathrm{~nm}$ particle size is sufficiently small to keep scattering low, an important feature for our DBRs. The TEM images and discussion can be found in the Supplementary Materials Section 1.4.

\subsection{Device design and optimisation}

Having established the optical properties of our multilayer thin films DBR materials, we set about building the design of our LSC-DBR combination.

As proof-of-concept we chose Lumogen Red 305 as the fluorophore. Lumogen is a widely commercially available fluorescent dye, with high quantum yield $(0.985 \pm 0.05)$. The high overlap between absorption and emission spectra also allows us to investigate the effects of the wavelength selective mirror on re-absorption losses, and how re-absorption effects LSC efficiency in the presence of DBRs. Lumogen is also easily integrated into a flexible PDMS host matrix, using the recipes and methods described in the methods section.

The presence of a back reflector is required, as if one is not used any light reflected off the DBR at near to normal angles of incidence is highly likely to escape out of the opposite side of the LSC. In this study, as a back reflector we used a nonscattering silver mirror in order to isolate the effects of a single DBR. Silver was chosen for its high reflectance across the visible spectrum. The use of a back reflector is not always appropriate as device transparency may be desired. In this case alternative configurations may be more suitable, such as the use of DBRs on both sides of the LSC, or cascade LSC-DBR stacks, each used to harvest particular segments of the solar spectrum [37]. 
An ideal wavelength selective mirror has high transmittance in the spectrum where the fluorophore absorbs, and a high reflectance in that emission range of the fluorophore. The aim is to maximise internal and external optical efficiencies, $\eta_{\text {int }}$ and $\eta_{\text {ext }}$, whilst minimising quantum yield losses and escape cone losses. Definitions for these efficiency metrics and losses can by found in the methods sections.

We can design and predict the optical properties of the multilayer thin films using a Transfer Matrix Model [27], making use of the refractive indices of our hPDMS and $\mathrm{TiO}_{2}$ composite, as established in the previous subsection. We then combined the Transfer Matrix Model with our in-house Monte-Carlo methods ray tracing algorithm, which we have previously been shown to accurately predict LSC efficiency [38, 24, 39]. A combination of the simulation platform, and consideration of fabrication complexity were used to optimise a series of the DBR parameters to partner the Lumogen Red doped LSCs. We optimised parameters including the number of layers in the DBR and thickness of each layer. An in-depth discussion of these optimisations is in the Supplementary Materials Section 2. The resultant DBR reflectance spectrum, which has a peak reflectance of 0.9 , is shown overlayed with the Lumogen Red 305 absorption and emission spectra for reference in Figure $3 \mathrm{c}$.

\subsection{Fabricated Flexible Distributed Bragg Reflectors}

We have demonstrated the fabrication of flexible DBRs consisting of 15 alternating layers of our Ti-PDMS composite and hPDMS. In Figure 3 we show a comparison between theoretically predicted and experimentally measured spectra at normal incidence, showing excellent agreement for two different wavelength selective mirrors, indicative for the high level of control and film quality that we achieved. The DBRs we have demonstrated DBRs were designed for two different wavelengths, both with peak reflectances of 0.90 (Figure 3b) and 0.92 (Figure 3c) respectively. The latter is in agreement with our previously discussed design, optimised for Lumogen Red 305 devices.

Using an Imaging Sphere we measured the beam profile before and after reflection off a DBR sample. This gives us an indication of the level of scattering of the DBRs. When coupled with the LSCs, due to the desired waveguiding effect, the highest number of interactions between light and LSC will be in the reflectance band of the LSC. As such, we took these measurements at the peak reflectance wavelength of $670 \mathrm{~nm}$. As shown in the Supplementary Materials Section 3.1, the beam profiles after reflection off an optically flat silicon wafer and reflection off a DBR are extremely similar. By calculating the de-convolution of the two beam profiles, as shown in Figure 3d, we derive the point-spread function of the DBR. We saw that 90 $\%$ of the point spread function falls within half a degree from $\theta=0$. Given the resolution $\left(0.5^{\circ}\right)$ and relative error $( \pm 5 \%)$ of the measurement equipment, the sample can be considered scattering free within the error of our experiment.

In order to demonstrate the uniformity of the DBR reflectances, we measured the normal reflectance of one of our DBRs on 9 different points in a $3 \times 3$ grid, with $2.5 \mathrm{~cm}$ spacing between each point on the grid, a map of which is shown in the Supplementary Materials Section 3.2. In Figure 3e we show the mean normal reflectance as a function of wavelength (blue) as well as two lines separated by one standard deviation from the mean. The average standard deviation across the entire measured spectrum is 0.036 , showing that the sample exhibits highly uniform reflectance throughout.

We have further characterised the performance of the DBRs, demonstrating both resistance to mechanical bending, and the independance of the reflectance at a range of temperatures $(25$ ${ }^{\circ} \mathrm{C}$ to $50{ }^{\circ} \mathrm{C}$ ). These studies can be found in the Supplementary Materials Section 3.2. Attempts to pull off the DBR from the PDMS body with tweezers failed, whilst a study in which we try to delaminate the DBR from the PDMS with scotch tape shows negligible change in reflectance, demonstrating the strong adhesion we achieve between layers and to the DBR and the PDMS substrate.

\subsection{Luminescent solar concentrator efficiency}

We fabricated three comparable LSCs in order to demonstrate the effects of our DBRs. One sample with just a standalone LSC (Sample A), one with a silver-mirror (Sample B), and finally one with both a silver mirror and our DBR (Sample C). The silver mirrors are deposited using thermal evaporation, and then encapsulated within PDMS. The measured and expected reflectances of the silver mirrors can be found in the Supplementary Materials Section 4.2.

To demonstrate the effect of our DBR we investigated the fates of photons once they are absorbed, allowing us to understand the waveguiding efficiency, as well as the effects of absorption losses. We used three metrics to quantify the waveguiding performance: internal optical efficiency, quantum yield losses and escape cone losses.

Figure 4 a shows a comparison between the simulated and measured photon fates for our devices when excited with a beam with centre-wavelength $504 \mathrm{~nm}$ and a spectral full-width half maximum of $20 \mathrm{~nm}$.

A key indicator of the effectiveness of our DBR devices is the reduction of escape cone losses. The observed escape cone losses in Sample A is 0.43 . This is reduced to 0.38 after the addition of the silver back reflector. Furthermore we saw a significant reduction to 0.31 with the DBR added, corresponding to a $28 \%$ reduction of escape cone losses relative to Sample A.

We saw a rise of the internal optical efficiency from 0.49 for Samples A and B respectively to 0.54 with the presence of the DBR in Sample C, an increase of $10 \%$. The presence of additional re-absorption losses can be used to account for the difference between the additional internal efficiency and the decrease in escape cone losses. The quantum yield losses increase from 0.08 to 0.12 and 0.15 across the three devices respectively. Whilst in this case, the contribution of the DBR to the LSC's efficiency is diminished by these additional re-absorption losses, it is not an indicator that the DBR is not performing as expected, as additional absorption losses are predicted by the Monte-Carlo model.

The internal optical efficiency, $\eta_{\text {int }}$ is a key parameter which shows how efficiently photons are guided towards the edges once they have been absorbed, but does not show the full story. 


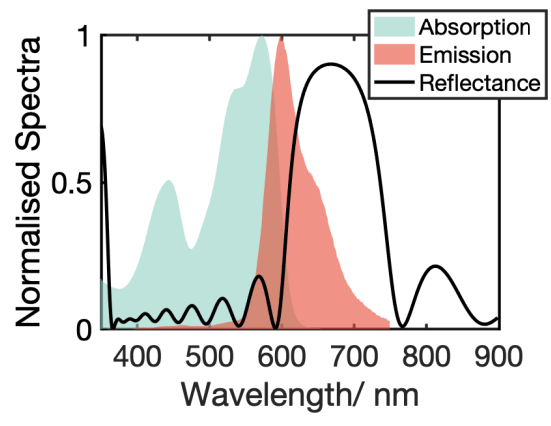

(a)

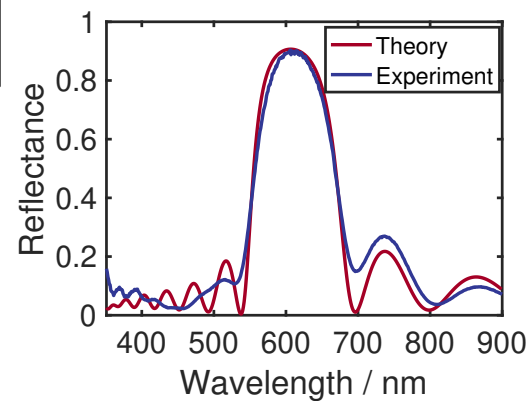

(b)

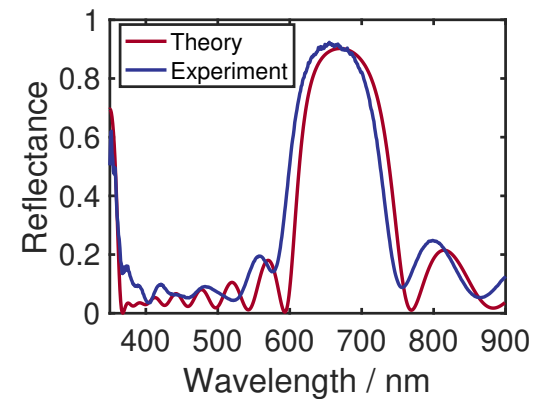

(c)

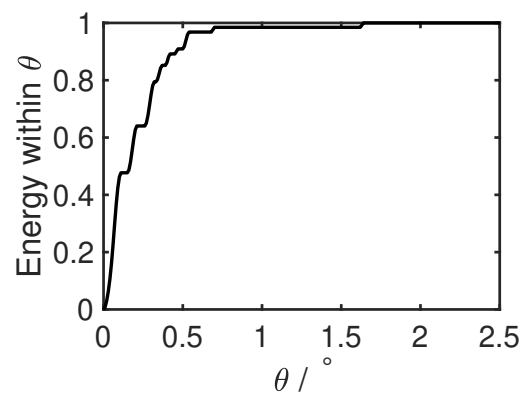

(d)

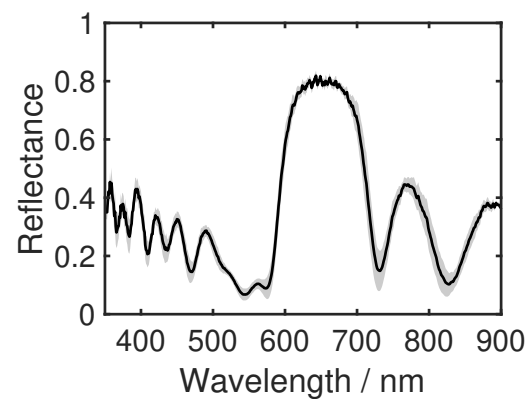

(e)

Figure 3: a) Simulated normal reflectance of DBR design (black) optimised for Lumogen Red 305. Also shown is the absorption (green) and emission (red) spectra of Lumogen Red 305. A comparison of the predicted theoretical (red) and measured experimental (blue) reflection spectra of fabricated flexible wavelength selective mirrors designed for peak reflectance at b) $605 \mathrm{~nm}$ and c) $670 \mathrm{~nm}$. d) Angular profile of the spread of the beam as a function of $\theta$ after reflection of the DBR. e) Measured reflectance of a DBR sample on $\mathrm{Si}$ as a function of wavelength. The mean values (black) of 9 measurements on the sample. Grey shading indicates a $1 \sigma$ error.

The external optical efficiency, $\eta_{\text {ext }}$ takes into account all photons that reach the LSC, including those that are reflected off the device, or are not absorbed within the host matrix. Using our simulation platform we calculate the external optical efficiency, shown in Table 1. In this case the values are normalised for the solar spectrum using the AM1.5 standard, bound by $300 \mathrm{~nm}$ and $660 \mathrm{~nm}$, the absorption range of Lumogen Red 305.

As Table 1 shows, despite just a $10 \%$ increase of internal optical efficiency from the LSC only device to the full device, we expect a $27 \%$ increase in external optical efficiency. This is due to the effects of additional absorption caused by the silver back reflector and the combination of this with the additional internal efficiency due to the DBR. The optical concentration factors, also defined in the Methods section, are shown in Table 1. These also take into account the geometric gain of the samples (12.5) and are adjusted for spectra across the entirety of AM1.5.

\begin{tabular}{llll}
\hline Device & $\eta_{\text {int }}$ & $\eta_{\text {ext }}$ & $\mathbf{C}_{F O}$ \\
\hline $\mathrm{A}$ & 0.52 & 0.33 & 3.34 \\
$\mathrm{~B}$ & 0.53 & 0.39 & 3.95 \\
$\mathrm{C}$ & 0.57 & 0.42 & 4.25 \\
\hline
\end{tabular}

Table 1: Internal $\eta_{\text {int }}$, external $\eta_{\text {ext }}$ optical efficiencies and concentration factors, $\mathrm{C}_{F O}$ as simulated for three comparable devices for values of AM1.5 between $300 \mathrm{~nm}$ and $660 \mathrm{~nm}$.

Integrating sphere measurements demonstrate the various photon fates of light once absorbed by the devices, which is independent of incident photon wavelength due to the KashaVavilov rule [40], however it is also important to measure the performance across the entire solar spectrum. For this we have taken device measurements using a solar simulator, when coupled to a commercially available solar cell. We performed comparative IV measurements using a solar simulator system for the three devices (A, B and C). As proof of concept, the devices were coupled, on one side, to a poly-crystalline silicon (pSi) solar cell module (RVFM-37038, Rapid Online). We used index matching fluid (Cargille, $\mathrm{n}=1.56$ ) to reduce interfacial reflection losses. We masked the solar cell to leave an open aperture with active area $5 \mathrm{~cm} \times 0.4 \mathrm{~cm}$. The exposed area of the solar cell module had an efficiency of $17 \%$ (JV measurement shown in Supplementary Materials Section 4.2). Our JV measurements, shown in Figure $4 \mathrm{~b}$ show an improvement in the short circuit current density from $5.4 \mathrm{mAcm}^{-2}$ for the plain LSC device, to $6.8 \mathrm{mAcm}^{-2}$ and $8.1 \mathrm{mAcm}^{-2}$ for the device with silver mirror, and DBR respectively. The device opticalto-electrical power conversion efficiencies were measured to be $2.3 \%, 2.8 \%$ and $3.2 \%$ for the LSC, LSC + Mirror and LSC + Mirror + DBR devices. This corresponds to a relative improvement of $22 \%$ from Sample A to Sample B, and a further $14 \%$ improvement from Sample B to Sample C. However the power conversion efficiencies in this case are highly dependant on the solar-cell unit used, and the coupling to the LSCs, which in this proof-of-concept study have not been optimised to these particular LSCs. Another useful benchmark is the ratio of the 


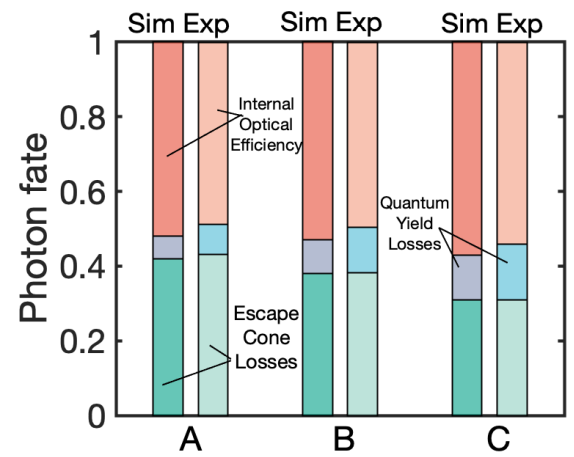

(a)

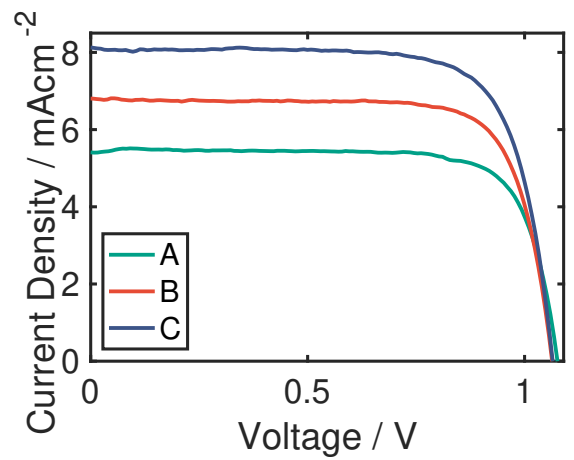

(b)

Figure 4: a) A comparison between the simulated (left of pairings) and experimental (right of pairings) photon fates for Samples A (LSC only), B (LSC + mirror) and C (LSC + Mirror + DBR) (from left to right). Quantum yield losses are depicted in blue, escape cone losses in green and internal optical efficiency in red. b) Measured Current density - Voltage sweep for Samples A, B and C coupled to $\mathrm{p}$-Si solar cell module.

power of the LSC devices to that of the stand alone solar-cell module, measured in the solar simulator. In this case, the ratios are 1.6, 2.1 and 2.4 for the three devices respectively. In practise this means that we are achieving a concentration equivalent to the direct exposure of 2.4 times the area of our solar-cell module. The difference between devices would further increase with larger geometric gain, as longer optical paths will result in a further increase of escape cone losses, which are more prominent in the case of devices without DBRs.

\subsection{Effects of curvature}

A key aspect of the viability of these devices is their performance under curvature. Figure 5 shows the decay in internal optical efficiency, $\eta_{\text {int }}$ as the radius of curvature is decreased for two devices: one device with LSC and mirror; and one with LSC, mirror and DBR. We show a comparison between our measurements, taken using the same integrating sphere setup as previously described, and our Monte-Carlo model.

We observed that the decrease in performance, from flat to curved, is lower for the device with the DBR at all curvatures, demonstrating a decoupling of LSC performance curvature. Our simulations show that the performance drop due to curvature is 3 times faster for a device without the DBR than one with a DBR. This can be attributed to the decrease in escapecone losses induced by the DBR, which is maintained with cur-

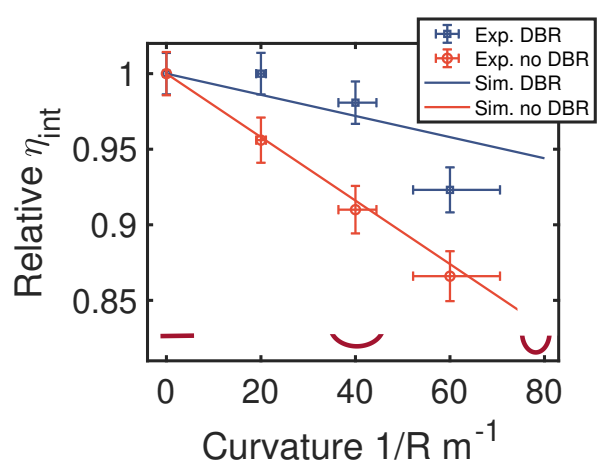

Figure 5: A comparison between simulated (lines) and measured (error bars) values of internal optical efficiency $\eta_{\text {int }}$ for a device with a DBR (red) and without a DBR (blue) as a function of curvature $(1 / R)$ where $R$ is the radius of curvature of the device. The values are normalised to the performance when flat.

vature due to the low curvature dependence of the DBR peak reflectance on curvature, as demonstrated in the Supplementary Materials Section 3.2. Whilst the trend of internal optical efficiencies follows the simulated values well for large radii of curvature, the accuracy of our experimental measurements is susceptible to increased errors at very small radii of curvature which we attribute to an observed buckling of the silver mirror at tight curvatures, resulting in a reduction in its reflectance. This was confirmed experimentally with repeated bending tests of standalone mirrors (results not shown). However, this is only evident at curvatures of $1 / \mathrm{R}>50 \mathrm{~m}^{-1}(\mathrm{R}<0.02 \mathrm{~m})$, and so does not apply to most applications of interest which would usually have curvatures $1 / \mathrm{R}<25 \mathrm{~m}^{-1}$. For reference, the radius of curvature of a watch strap is $\approx 0.04 \mathrm{~m}$, for which the equivalent $1 / \mathrm{R}=25 \mathrm{~m}^{-1}$. Furthermore, for larger applications such as buildings, bending a $1 \mathrm{~m}^{2}$ square device to a semi-circular shape gives a radius of curvature of $\approx 0.3 \mathrm{~m}$. For a table of some typical radii of curvature, refer to Supplementary Materials Section 6. The y-error bars signify 1 standard-deviation of the repeat experimental measurements of internal optical efficiency across the devices at all curvatures. The x-error bars are based on a $1 \mathrm{~cm}$ variation of radius of curvature across the devices when curved for the measurements due to the curvature only being applied at two points on the device. Reducing the dependence of LSCs on curvature shows the flexible LSC-DBR device makes the technology more suitable for use on curved building facades and alternate applications such as boat sails and wearable technologies.

\subsection{Scaling and potential}

In this section we investigate the effects of LSC size scaling as well as the effects of stokes shift on the performance of our LSC devices and their combination with the DBRs. In the previous sections we have shown both experimentally and by simulation that our flexible DBRs can be used to enhance the performance of devices with dimensions of $5 \mathrm{~cm} \times 5 \mathrm{~cm}$, however it is of interest to explore the performance of such devices for larger collection sizes. Using our Monte-Carlo model we explored how the performance of these devices is affected by 
size, by scaling the lateral size of the LSCs, whilst maintaining a thickness of $0.5 \mathrm{~cm}$. Figure 6 a shows the simulated performance of two devices, one with the LSC + silver mirror (orange) and one with the LSC + DBR + silver mirror (blue), both in a flat configuration. The enhancement is the relative performance of the devices in comparison with an LSC device. In the case of these simulations all the devices are doped with Lumogen Red 305 at a fixed concentration of $10^{-4} \mathrm{M}$, in a PDMS host matrix. Our simulations show that the relative performance of the device with the DBR is continually increased as lateral size is increased. This is due to the reduction of escape cone losses associated with the DBR reducing the dependence of LSC to lateral size. DBRs can therefore help with up-scaling issues associated with LSC manufacture. The resultant devices for dimensions $1 \mathrm{~m} \times 1 \mathrm{~m} \times 0.5 \mathrm{~cm}$ display an enhancement for $\eta_{\text {ext }}^{\prime}$ across AM1.5 from $5.0 \%$ for LSC + mirror to $5.7 \%$ with the addition of the DBR. This is equivalent to the amount of light being delivered to the edges of the LSC for the $1 \mathrm{~m}^{2}$ panel rising from $50 \mathrm{~W}$ to $57 \mathrm{~W}$. Further simulations show that in the case that $1 \mathrm{~m}^{2}$ the devices are bent to a semi-circular configuration we see a curvature induced performance drop of $0.5 \%$ in absolute terms to $\eta_{\text {ext }}^{\prime}=4.5 \%$ for the LSC with silver mirror but just a $0.1 \%$ drop to $\eta_{e x t}^{\prime}=5.6 \%$ in the case with the DBR. This means that in the case of a curved configuration for $1 \mathrm{~m}^{2}$ devices we have an enhancement from $45 \mathrm{~W}$ to $56 \mathrm{~W}$, a $24 \%$ enhancement in relative terms.
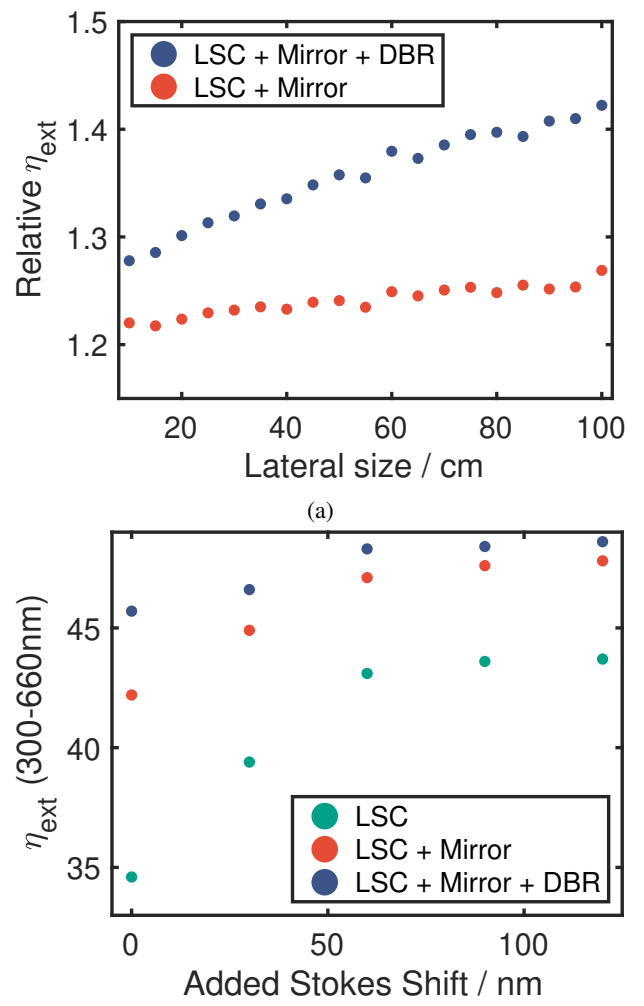

(b)

Figure 6: a) Enhancement of devices in flat configuration (relative performance) as a function of lateral size for two devices: with a DBR and silver mirror (blue) and with just a silver mirror (orange). b) External optical efficiency as a function of Stokes Shift for three devices: LSC Only (green), LSC + Silver Mirror (orange) and LSC + Silver Mirror + DBR (blue).
So far we have simulated the performance of such devices on Lumogen Red 305, however as discussed in the introduction, most highly efficient LSCs in recent publications are based on materials with less overlap between absorption and emission spectra. To explore the effects of spectral overlap we simulated the performance a set of devices whilst artificially modifying the Stokes shift. Figure $6 \mathrm{~b}$ shows the external optical efficiencies $\eta_{\text {ext }}$ for a series of $1 \mathrm{~m} \times 1 \mathrm{~m}$ by $0.5 \mathrm{~cm}$ in size, for 3 comparable sets of devices. All 3 sets of devices are doped with Lumogen Red, however at each data point we add $30 \mathrm{~nm}$ of Stokes shift to all reemitted light, thus reducing the amount of spectral overlap. In order to derive a situation in which we eventually have no spectral overlap we also artificially shorten the long emission tail (below $520 \mathrm{~nm}$ before additional stokes shift, as discussed in the supplementary materials Section 4.3). In the case of the LSC only device (green), as expected the efficiency increases with reduced spectral overlap, however approaches saturation as reabsorption losses are eliminated. In the case of the devices enhanced by the DBR (red), the boost to optical efficiency relative to devices with a silver mirror but no DBR (orange) is gradually reduced as Stokes shift is increased. This is due to the fact the modelled DBRs have peak reflectance is $\approx 0.9$ and not unity. The DBRs are used to trap light which originally falls within the escape cone loss of the host matrix. In these cases the light is incident on the DBR at near normal angles of incidence, and so if reflected will undergo a series of reflections off top and bottom surfaces of the LSC. Due to the non perfect nature of both top and bottom mirrors, a proportion of light will be lost upon each reflection if not otherwise redirected. For large devices as modelled here, the light is therefore unlikely to reach the edges as desired. In this case reabsorption is beneficial, as there becomes a chance that the oscillating light will be reabsorbed and redirected for a more direct path towards the edges. This does not however limit the use of DBRs to fluorophores with significant spectral overlap as improved enhancement can be achieved with DBRs of higher peak reflectance. As the number of layers used in the DBR increases, the peak reflectance follows, and so the loss at each interface is reduced. This increases the viable size of LSC for which DBRs provide a significant boost in efficiency.

\section{Conclusions}

We have demonstrated for the first time an all flexible LSCDBR combination formed entirely of silicone based components. By creating DBRs comprised of hPDMS and a titaniaPDMS composite we achieve devices with strong adhesion and flexibility throughout. The excellent control we exhibit in the optical properties and thickness of the layers in the thin films allows us to design flexible DBRs with high and precisely engineered reflectances. Our DBRs display high uniformity, low scattering and show little dependence on temperature and bending. We have demonstrated them on 3 inch wafer scale, using scalable solution based processes. Whilst our DBRs were fabricated using spin-coating, the solution based recipe opens opportunity for similar results with other industrially available techniques such as spray-coating or dip-coating 
[41]. Our DBRs could be used as standalone strain or solvent sensing as shown in previous demonstrations of flexible DBRs $[42,43,44,45,46]$.

In turn, we used the DBRs to enhance the efficiency of Lumogen Red 305 doped PDMS based LSCs. The DBRs have reduced escape cone losses by over a quarter for a $5 \times 5 \times 0.4$ $\mathrm{cm}$ device, resulting in a $10 \%$ increase in internal optical efficiency. We've shown that with the reduction of escape cone losses we lower the dependence of efficiency on curvature, rendering the devices more suitable for use on curved facades and flexible applications such as boat sails and wearable technologies.

Furthermore, with our simulation platform we have shown that the enhancement provided by the DBRs is increased for larger collection areas, giving an enhancement in external optical efficiency, $\eta_{\text {ext }}^{\prime}$ from $5.0 \%$ to $5.7 \%$ in a flat configuration and $\eta_{\text {ext }}^{\prime}$ from $4.5 \%$ to $5.6 \%$ in a curved configuration $(1 / \mathrm{R}=$ $3.1 \mathrm{~m}^{-1}$ ), a $24 \%$ relative improvement. The consequence of which is that we have essentially decoupled the performance of the LSC from curvature for most commercially relevant applications.

\section{Materials and Methods}

A graphic summarising the fabrication steps of the LSCDBR combination can be found in the Supplementary Materials, Section 5 .

\subsection{Preparation of hPDMS solution}

hPDMS (GELEST) was made by mixing equal parts by weight of Part A (Base) and Part B (Crosslinker) and dissolving in ethyl-acetate $99.8 \%$ (anhydrous) (Sigma-Aldrich) into a round bottomed glass flask. We thoroughly mixed the solution until entirely dissolved. The percentage weight ratio of hPDMS to ethyl-acetate was varied according to desired thickness. A percentage weight of $\approx 4 \%$ results in thicknesses of $\approx 100 \mathrm{~nm}$ when spin coated at spin speeds of 3000-5000 rpm at room temperature and humidity of $\approx 50 \%$.

\subsection{Preparation of PDMS-TiO ${ }_{2}$ composite solution}

Polydimethylsiloxane, hydroxy terminated, M.W. 4200 (Alfa Aesar) was dissolved in ethyl acetate $99.8 \%$ (anhydrous) (Sigma-Aldrich) at a ratio of $6.7 \%$ by weight into a round bottomed glass flask. Acetic acid (Sigma-aldrich) was added at a concentration of $2 \%$ by weight. We varied this according to desired optical properties according to the main discussion section. $0.5 \%$ by weight of BYK-378 surfactant was added. We then sealed the flask with a rubber septum and stirred for 5 minutes with a magnetic stirrer bar. We mixed Titanium (IV) isopropoxide (97\%, Sigma-aldrich) with ethyl acetate with a ratio of $27 \%$ by weight and slowly added by syringe through the rubber septum to the main solution. All percentages by weight given are relative to the total solution weight.

\subsection{Preparation of water-soluble sacrificial layer}

In order to achieve highly uniform films, a solid substrate is needed for spin-coating. 3-inch silicon wafers were used as our substrates, with a water soluble sacrificial layer, in this case Poly(vinyl) alchohol (PVA), MW 9,000-10,000, 80 \% hydrolyzed (Sigma-Aldrich), which was used due to its high solubility in water and resistance to ethyl acetate. PVA, $5 \%$ by weight, was dissolved in de-ionised water, which we left to stir with a magnetic stirrer bar at $90^{\circ} \mathrm{C}$ for 24 hours in order to achieve good dispersion. This was then deposited the sacrificial layer using spin-coating at 3000RPM, before baking on the hot-plate at $120^{\circ} \mathrm{C}$ for 2 minutes.

\subsection{Fabrication of multilayer structures}

Alternate layers of the hPDMS and the PDMS- $\mathrm{TiO}_{2}$ composite solutions were deposited using spin coating. For good quality films spin speeds of 3000-6000 RPM are recommended. Spin speeds were optimised for desired thicknesses [47]. Between depositions the samples were left to rest for excess solvent to evaporate. The resting time varies depending on laboratory humidity and temperature. Spin coating was done under a nitrogen environment. Short rest times can result in pinholes. Shortly before each deposition the samples were exposed to oxygen plasma for one minute to activate the surfaces of each layer for good adhesion and wetting. Making the outside layers of the multilayer structure (neighbouring the PVA and LSC) consist of the $\mathrm{TiO}_{2}$ composite gives the highest contrast in refractive index between the DBR and its surrounding layers.

\subsection{Fabrication of LSC}

In order to achieve the maximum possible adhesion between DBR and LSC, the LSCs were cured to the DBR. The LSCs were then fabricated following a recipe for PDMS from a previous paper [5]. The desired luminophore, in this case Lumogen 305, was diluted in ethyl acetate and sonicated in chilled water $\left(\approx 5^{\circ} \mathrm{C}\right)$. Meanwhile, the PDMS mixture (Sylgard-184, Dow) was prepared. The elastomer (Part A) was mixed with the curing agent (Part B) in a 9:1 ratio. The dye-solvent solution was added to the PDMS in the desired concentration. The whole solution was then mixed for an hour in a chilled ultrasonic bath $\left(\approx 5^{\circ} \mathrm{C}\right)$. This was then degassed in a vacuum oven. The prepared DBR was then placed into a mould, and poured the PDMS-luminophore solution over the DBR, and allowed it to cure. The PDMS was then cured for 48 hours at room temperature, however can be accelerated with heat. Accelerating the curing process using heat can result in a reduction in the mechanical properties of the sample [48].

\subsection{Dissolution of sacrificial layer}

Once the PDMS is sufficiently cured, the sacrificial layer was dissolved by taking the sample out of the mould and leaving in a chilled ultra-sonic bath $\left(\approx 5^{\circ} \mathrm{C}\right)$. We left the sacrificial layer in the ultrasonic bath until the device separated from the silicon wafer. In some cases this can take multiple hours, with no noticeable damage to the DBRs, demonstrating the strong adhesion of the DBRs to the PDMS and of individual DBR layers. 


\subsection{Deposition of silver mirror}

A $250 \mathrm{~nm}$ silver mirror was depsoited using thermal evaporation (Edwards, A308 FL400) after exposing the samples to oxygen plasma for 1 minute. The the whole device was then encapsulated with PDMS in order to protect the mirror from damage.

\subsection{Optical Characterisation}

The internal optical efficiency, quantum yield losses and escape cone losses of our LSC devices were measured with an integrating sphere setup using the method described in [39]. The normal reflectance measurements were performed using a white halogen light source (20W, HL-2000, Ocean Optics), fiber optic reflection probe bundle setup (Ocean Optics) and spectrophotometer (Ocean Optics).A calibrated reflectance standard (STAN-SSL Ocean Optics) was used as reference. Temperature dependent measurements were performed by attaching the sample to a heating stage and calibrated thermocouple. Ellipsometric measurements were taken using a Semilab SE-2000 spectroscopic ellipsometer. Fitting of ellipsometric constants $\Delta$ and $\Psi$ was performed in Semilab Spectroscope Ellipsometry Analyzer software (SEA, Semilab). Attenuated Total Reflectance - Fourier Transform Infrared Spectroscopy (ATR-FTIR) was used to obtain the FTIR absorbance spectra of the PDMS-OH and PDMS-TiO 2 composites using an FTIR spectrophotometer (Shimadzu, IRPrestige-21). Scattering analysis measurements were taken using an Imaging-Sphere (IS-SA, Radiant Zemax) at an angle of $8^{\circ}$ from normal incidence. IV measurements were performed using a solar simulator system (LS09505, LOT Oriel).

\subsection{Materials Characterisation}

Transmission Electron Microscopy (TEM): Conventional TEM images were acquired on a (JEOL 2010, JEOL) TEM operating at $200 \mathrm{kV}$. Image collection and processing was performed on a CCD with Gatan Digital Micrograph software and ImageJ.

X-ray Photoelectron Spectroscopy (XPS): XPS analysis was carried out using a K-alpha photoelectron spectrometer (Thermo Scientific) with monochromatic $\mathrm{Al}-\mathrm{K} \alpha$ radiation. Peak positions were calibrated to carbon $(284.8 \mathrm{eV})$.

\subsection{Modelling}

Our computational platform used to predict the performance of LSCs is a Monte-Carlo ray-tracing model, which has provided accurate predictions of device efficiencies in our previous studies [39]. Light in the LSC was modelled as a ray propagating throughout a medium, which is reflected or transmitted at the interfaces of the LSC. The probabilities of reflection and transmission were calculated according to Fresnel's laws in the case of LSC-air interfaces. In the case of interfaces involving the silver mirrors or DBRs, reflectance and transmittance coefficients are generated using a Transfer Matrix Model (TMM) used to calculate the optical properties of layer media, taking into account thin-film interference [27]. Beer-lambert law was used to generate the optical path before a photon is absorbed within the LSC medium, using absorption cross-sections measured using a UV-Vis spectrophotometer (Shimadzu UV-1800). The probability of re-emission is based on the fluorophore's quantum yield, which is experimentally measured, and adjusted to take into account device dimensions and concentration. The wavelength of the re-emitted photon is based on a probability distribution sampled from the emission spectrum of the fluorophore, which is experimentally measured using time correlated single photon count spectroscopy (TCSPC) (LifeSpec-ps, Edinburugh Instruments).

\subsection{Figures of merit}

In this subsection we define the figures of merit used in the paper. We define internal optical efficiency, $\eta_{\text {int }}$, as the number of photons successfully guided to the edges divided by the total number of photons absorbed by the LSC device. Quantum yield losses are losses caused by non unity quantum yields of the fluorophores and can be defined by the total number of photons absorbed by the device but not reemitted divided by the total number of photons absorbed by the device. Escape cone losses are where light escapes the larger faces of the LSC and does not reach the edges, and can be defined as the number of photons that are refracted out of the two larger faces of the LSC divided by the number of incident photons absorbed. External optical efficiency is derived, $\eta_{\text {ext }}=A \eta_{\text {int }}$ where $A$ is the proportion of incident photons absorbed, taking into account light that is reflected off the device and light that is transmitted through the device. The optical concentration factor, $C_{O F}$ is the quantity of optical flux that is successfully guided to the edges divided by the total incident flux, multiplied by the geometric gain factor, $G$. $G$ is the ratio of collection surface area to concentration surface area. The optical concentration factor, $C_{F O}$ can be calculated by $C_{F O}=\eta_{e x t}^{\prime} G$ where $\eta_{e x t}^{\prime}=\eta_{\text {ext }} K$. In this case $K$ is a correction factor adjusting $\eta_{\text {ext }}$ from ratio of photons into a ratio of fluxes by taking into account the energy of each photon. In the case of the devices coupled to solar cells, we quantify the device optical-to-electrical power conversion, $\eta_{O E}=V_{O C} I_{S C} F F / P_{i n} . V_{O C}$ is the open circuit voltage. $I_{S C}$ is the short circuit current. FF is the fill factor and $P_{i n}$ is the incident power.

\section{Acknowledgements}

We would like to thank the UK Engineering and Physical Sciences Research Council (EPSRC) for a Doctoral training award grant no: 1632762 . The work was supported by a H2020 European Research Council (ERC) starting grant "IntelGlazing" grant no: 679891. T.J.M would like to thank the Ramsay Memorial Trust and the Royal Commission for the Exhibition of 1851 for their financial support.

\section{Author contributions statement}

M.P. and I.P. conceived the experiments. M.P. developed and fabricated the samples and performed characterisation experiments. M.P. developed the modeling system. T.J.M. performed 
surface chemistry experiments. C.S. and M.P. performed ellipsometric measurements and fitting. M.P. T.S.R. and J.S. contributed to the development of the PDMS - $\mathrm{TiO}_{2}$ composite. T.L. developed and performed silver mirror deposition. S.G., I.P.P and I.P. facilitated the work and engaged with experiment design. All authors reviewed and contributed to the manuscript.

\section{Additional information}

The authors declare no competing interests.

\section{References}

[1] S. F. Correia, P. P. Lima, P. S. André, M. R. S. Ferreira, L. A. D. Carlos, High-efficiency luminescent solar concentrators for flexible waveguiding photovoltaics, Solar Energy Materials and Solar Cells 138 (2015) 51-57. doi:10.1016/j.solmat.2015.02.032.

[2] J. Yoon, L. Li, A. V. Semichaevsky, J. H. Ryu, H. T. Johnson, R. G. Nuzzo, J. A. Rogers, Flexible concentrator photovoltaics based on microscale silicon solar cells embedded in luminescent waveguides, Nature Communications 2 (2011) 343. doi:10.1038/ncomms1318.

[3] M. Buffa, S. Carturan, M. G. Debije, A. Quaranta, G. Maggioni, Solar Energy Materials \& Solar Cells Dye-doped polysiloxane rubbers for luminescent solar concentrator systems, Solar Energy Materials and Solar Cells 103 (2012) 114-118. doi:10.1016/j.solmat.2012.04.019.

[4] C. H. Chou, M. H. Hsu, F. C. Chen, Flexible luminescent waveguiding photovoltaics exhibiting strong scattering effects from the dye aggregation, Nano Energy 15 (2015) 729-736. doi:10.1016/j.nanoen.2015.06.001.

[5] C. Tummeltshammer, A. Taylor, A. J. Kenyon, I. Papakonstantinou, Flexible and fluorophore-doped luminescent solar concentrators based on polydimethylsiloxane, Optics Letters 41 (2016) 713. doi:10.1364/OL.41.000713.

[6] I. A. Carbone, K. R. Frawley, M. K. McCann, Flexible, Front-Facing Luminescent Solar Concentrators Fabricated from Lumogen F Red 305 and Polydimethylsiloxane, International Journal of Photoenergy 2019 (2019) 1-9. doi:10.1155/2019/8680931.

[7] I. Meazzini, N. Willis-Fox, C. Blayo, J. Arlt, S. Clément, R. C. Evans, Targeted design leads to tunable photoluminescence from perylene dicarboxdiimide-poly(oxyalkylene)/siloxane hybrids for luminescent solar concentrators, Journal of Materials Chemistry C 4 (2016) 4049-4059. doi:10.1039/c5tc03952e.

[8] A. Kaniyoor, B. Mckenna, S. Comby, R. C. Evans, Design and Response of High-Efficiency, Planar, Doped Luminescent Solar Concentrators Using Organic-Inorganic Di-Ureasil Waveguides, Advanced Optical Materials 4 (2016) 444-456. doi:10.1002/adom.201500412.

[9] F. Meinardi, S. Ehrenberg, L. Dhamo, F. Carulli, M. Mauri, F. Bruni, R. Simonutti, U. Kortshagen, S. Brovelli, Highly efficient luminescent solar concentrators based on earth-Abundant indirectbandgap silicon quantum dots, Nature Photonics 11 (2017) 177-185. doi:10.1038/nphoton.2017.5.

[10] Y. Guo, Y. Chen, E. Wang, M. Cakmak, Roll-to-roll continuous manufacturing multifunctional nanocomposites by electric-field-assisted "z" direction alignment of graphite flakes in poly(dimethylsiloxane), ACS Applied Materials and Interfaces 9 (2017) 919-929. doi:10.1021/acsami.6b13207.

[11] R. R. Søndergaard, M. Hösel, F. C. Krebs, Roll-to-Roll fabrication of large area functional organic materials, Journal of Polymer Science, Part B: Polymer Physics 51 (2013) 16-34. doi:10.1002/polb.23192.

[12] I. Papakonstantinou, C. Tummeltshammer, Fundamental limits of concentration in luminescent solar concentrators revised: the effect of reabsorption and nonunity quantum yield, Optica 2 (2015) 841. doi:10.1364/OPTICA.2.000841.

[13] G. Smestad, H. Ries, R. Winston, E. Yablonovitch, The thermodynamic limits of light concentrators, Solar Energy Materials 21 (1990) 99-111. doi:10.1016/0165-1633(90)90047-5. arXiv: 1303.4604.
[14] M. Sharma, K. Gungor, A. Yeltik, M. Olutas, B. Guzelturk, Y. Kelestemur, T. Erdem, S. Delikanli, J. R. McBride, H. V. Demir, Near-Unity Emitting Copper-Doped Colloidal Semiconductor Quantum Wells for Luminescent Solar Concentrators, Advanced Materials 29 (2017) 1-10. doi:10.1002/adma.201700821.

[15] F. Meinardi, A. Colombo, K. A. Velizhanin, R. Simonutti, M. Lorenzon, L. Beverina, R. Viswanatha, V. I. Klimov, S. Brovelli, Large-area luminescent solar concentrators based on Stokes-shift-engineered nanocrystals in a mass-polymerized PMMA matrix, Nature Photonics 8 (2014) 392-399. doi:10.1038/nphoton.2014.54.

[16] H. Li, K. Wu, J. Lim, H. J. Song, V. I. Klimov, Doctor-blade deposition of quantum dots onto standard window glass for low-loss largearea luminescent solar concentrators, Nature Energy 1 (2016) 16157. doi:10.1038/nenergy.2016.157.

[17] D. A. Hanifi, N. D. Bronstein, B. A. Koscher, Z. Nett, J. K. Swabeck, K. Takano, A. M. Schwartzberg, L. Maserati, K. Vandewal, Y. van de Burgt, A. Salleo, A. P. Alivisatos, Redefining near-unity luminescence in quantum dots with photothermal threshold quantum yield, Science 363 (2019) 1199-1202. doi:10.1126/science.aat3803.

[18] S. K. Hill, R. Connell, C. Peterson, J. Hollinger, M. A. Hillmyer, U. Kortshagen, V. E. Ferry, Silicon Quantum Dot-Poly(methyl methacrylate) Nanocomposites with Reduced Light Scattering for Luminescent Solar Concentrators, ACS Photonics 6 (2019) 170-180. doi:10.1021/acsphotonics.8b01346.

[19] M. Wei, F. P. G. de Arquer, G. Walters, Z. Yang, L. N. Quan, Y. Kim, R. Sabatini, R. Quintero-Bermudez, L. Gao, J. Z. Fan, F. Fan, A. GoldParker, M. F. Toney, E. H. Sargent, Ultrafast narrowband exciton routing within layered perovskite nanoplatelets enables low-loss luminescent solar concentrators, Nature Energy (2019). doi:10.1038/s41560-018-0313$\mathrm{y}$.

[20] K. Nikolaidou, S. Sarang, C. Hoffman, B. Mendewala, H. Ishihara, J. Q. Lu, B. Ilan, V. Tung, S. Ghosh, Hybrid Perovskite Thin Films as Highly Efficient Luminescent Solar Concentrators, Advanced Optical Materials 4 (2016) 2126-2132. doi:10.1002/adom.201600634.

[21] H. Zhao, Y. Zhou, D. Benetti, D. Ma, F. Rosei, Perovskite quantum dots integrated in large-area luminescent solar concentrators, Nano Energy 37 (2017) 214-223. doi:10.1016/j.nanoen.2017.05.030.

[22] X. Luo, T. Ding, X. Liu, Y. Liu, K. Wu, Quantum-Cutting Luminescent Solar Concentrators Using Ytterbium-Doped Perovskite Nanocrystals, Nano Letters (2018) acs.nanolett.8b03966. doi:10.1021/acs.nanolett.8b03966.

[23] S. McDowall, T. Butler, E. Bain, K. Scharnhorst, D. Patrick, Comprehensive analysis of escape-cone losses from luminescent waveguides, Applied Optics 52 (2013) 1230-1239. doi:10.1364/AO.52.001230.

[24] M. Portnoi, C. Sol, C. Tummeltshammer, I. Papakonstantinou, Impact of curvature on the optimal configuration of flexible luminescent solar concentrators, Optics Letters 42 (2017) 2695. doi:10.1364/OL.42.002695.

[25] P. P. C. Verbunt, S. Tsoi, M. G. Debije, D. J. Boer, C. W. Bastiaansen, C.-W. Lin, D. K. G. de Boer, Increased efficiency of luminescent solar concentrators after application of organic wavelength selective mirrors, Optics Express 20 (2012) A655. doi:10.1364/oe.20.00a655.

[26] P. P. Verbunt, D. K. De Boer, D. J. Broer, M. G. Debije, Special dispersion chiral nematic reflectors for luminescent solar concentrators, 2015 IEEE 42nd Photovoltaic Specialist Conference, PVSC 2015 (2015). doi:10.1109/PVSC.2015.7355930.

[27] P. Yeh, Optical Waves in Layered Media, Wiley, Hoboken, NJ, 2005.

[28] R. Connell, V. E. Ferry, Integrating Photonics with Luminescent Solar Concentrators: Optical Transport in the Presence of Photonic Mirrors, Journal of Physical Chemistry C 120 (2016) 20991-20997. URL: http://pubs.acs.org/doi/abs/10.1021/acs.jpcc.6b03304. doi:10.1021/acs.jpcc.6b03304.

[29] R. Connell, C. Pinnell, V. E. Ferry, Designing spectrally-selective mirrors for use in luminescent solar concentrators, Journal of Optics (United Kingdom) 20 (2018). doi:10.1088/2040-8986/aaa074.

[30] H. J. Song, B. G. Jeong, J. Lim, D. C. Lee, W. K. Bae, V. I. Klimov, Performance Limits of Luminescent Solar Concentrators Tested with Seed/Quantum-Well Quantum Dots in a SelectiveReflector-Based Optical Cavity, Nano Letters 18 (2018) 395-404. doi:10.1021/acs.nanolett.7b04263.

[31] L. Xu, Y. Yao, N. D. Bronstein, L. Li, A. P. Alivisatos, R. G. Nuzzo, Enhanced Photon Collection in Luminescent Solar Concentrators 
with Distributed Bragg Reflectors, ACS Photonics 3 (2016) 278-285. doi:10.1021/acsphotonics.5b00630.

[32] N. D. Bronstein, Y. Yao, L. Xu, E. O’Brien, A. S. Powers, V. E. Ferry, A. P. Alivisatos, R. G. Nuzzo, Quantum Dot Luminescent Concentrator Cavity Exhibiting 30-fold Concentration, ACS Photonics 2 (2015) 15761583. doi:10.1021/acsphotonics.5b00334.

[33] G. Iasilli, R. Francischello, P. Lova, S. Silvano, A. Surace, G. Pesce, M. Alloisio, M. Patrini, M. Shimizu, D. Comoretto, A. Pucci, Luminescent solar concentrators: Boosted optical efficiency by polymer dielectric mirrors, Materials Chemistry Frontiers 3 (2019) 429-436. doi:10.1039/c8qm00595h.

[34] Corning, Corning SMF-28e Product information, 2007. URL: http://www.princetel.com/datasheets/SMF28e.pdf.

[35] A. Dalod, O. Grendal, A. Blichfeld, V. Furtula, J. Pérez, L. Henriksen, T. Grande, M.-A. Einarsrud, Structure and Optical Properties of TitaniaPDMS Hybrid Nanocomposites Prepared by In Situ Non-Aqueous Synthesis, Nanomaterials 7 (2017) 460. doi:10.3390/nano7120460.

[36] H. I. Elim, B. Cai, Y. Kurata, O. Sugihara, T. Kaino, T. Adschiri, A. L. Chu, N. Kambe, Refractive index control and rayleigh scattering properties of transparent TiO2 nanohybrid polymer, Journal of Physical Chemistry B 113 (2009) 10143-10148. doi:10.1021/jp902598f.

[37] M. J. Currie, J. K. Mapel, T. D. Heidel, S. Goffri, M. A. Baldo, Highefficiency organic solar concentrators for photovoltaics, Science 321 (2008) 226-228. doi:10.1126/science.1158342.

[38] C. Tummeltshammer, A. Taylor, A. J. Kenyon, I. Papakonstantinou, Homeotropic alignment and Förster resonance energy transfer: The way to a brighter luminescent solar concentrator, Journal of Applied Physics 116 (2014) 1-7. doi:10.1063/1.4900986.

[39] C. Tummeltshammer, A. Taylor, A. J. Kenyon, I. Papakonstantinou, Losses in luminescent solar concentrators unveiled, Solar Energy Materials and Solar Cells 144 (2016) 40-47. doi:10.1016/j.solmat.2015.08.008.

[40] J. R. Lakowicz, Principles of fluorescence spectroscopy, Springer US, Boston, MA, 2006. doi:10.1007/978-0-387-46312-4.

[41] D. Chen, Anti-reflection (AR) coatings made by sol-gel processes: A review, Solar Energy Materials and Solar Cells 68 (2001) 313-336. doi:10.1016/S0927-0248(00)00365-2.

[42] M. E. Calvo, H. Míguez, Flexible, adhesive, and biocompatible Bragg mirrors based on polydimethylsiloxane infiltrated nanoparticle multilayers, Chemistry of Materials 22 (2010) 3909-3915. doi:10.1021/cm1001016.

[43] M. Kolle, B. Zheng, N. Gibbons, J. J. Baumberg, U. Steiner, Stretchtuneable dielectric mirrors and optical microcavities, Optics Express 18 (2010) 4356. doi:10.1364/OE.18.004356.

[44] M. Kolle, A. Lethbridge, M. Kreysing, J. J. Baumberg, J. Aizenberg, P. Vukusic, Bio-inspired band-gap tunable elastic optical multilayer fibers, Advanced Materials 25 (2013) 2239-2245. doi:10.1002/adma.201203529.

[45] O. Sánchez-Sobrado, M. E. Calvo, H. Míguez, Versatility and multifunctionality of highly reflecting Bragg mirrors based on nanoparticle multilayers, Journal of Materials Chemistry 20 (2010) 8240-8246. doi:10.1039/c0jm01508c.

[46] M. E. Calvo, O. Sánchez Sobrado, G. Lozano, H. Míguez, Molding with nanoparticle-based one-dimensional photonic crystals: A route to flexible and transferable Bragg mirrors of high dielectric contrast, Journal of Materials Chemistry 19 (2009) 3144-3148. doi:10.1039/b902090j.

[47] D. B. Hall, P. Underhill, J. M. Torkelson, Spin coating of thin and ultrathin polymer films, Polymer Engineering and Science 38 (1998) 2039-2045. doi:10.1002/pen.10373.

[48] I. D. Johnston, D. K. McCluskey, C. K. Tan, M. C. Tracey, Mechanical characterization of bulk Sylgard 184 for microfluidics and microengineering, Journal of Micromechanics and Microengineering 24 (2014). doi:10.1088/0960-1317/24/3/035017. 